\title{
Artelogie
}

Recherche sur les arts, le patrimoine et la littérature de l'Amérique latine

$3 \mid 2012$

Image de la nation : art et nature au Chili

\section{L'empreinte de la nature sous le gouvernement militaire d'Augusto Pinochet (1973-1990) : quelques formulations à propos d'un art national}

Carine Lemouneau

\section{(2) OpenEdition}

Édition électronique

URL : https://journals.openedition.org/artelogie/7026

DOI : 10.4000/artelogie.7026

ISSN : 2115-6395

Éditeur

Association ESCAL

Référence électronique

Carine Lemouneau, «L'empreinte de la nature sous le gouvernement militaire d'Augusto Pinochet (1973-1990) : quelques formulations à propos d'un art national », Artelogie [En ligne], 3 | 2012, mis en ligne le 12 septembre 2012, consulté le 07 janvier 2022. URL : http://journals.openedition.org/ artelogie/7026 ; DOI : https://doi.org/10.4000/artelogie.7026

Ce document a été généré automatiquement le 7 janvier 2022.

Association ESCAL 


\section{L'empreinte de la nature sous le gouvernement militaire d'Augusto Pinochet (1973-1990) : quelques formulations à propos d'un art national}

\section{Carine Lemouneau}

\section{Introduction}

1 Difficile de se balader dans la capitale chilienne sans croiser au détour d'une rue l'imposante Cordillère des Andes. Si les hauteurs du parc Santa Lucía ou du Cerro San Cristóbal nous offrent un panorama exceptionnel, sa présence dans le quotidien des habitants se manifeste jusqu'aux couloirs du métro : suite au récent projet MetroArte ${ }^{1}$ lancé en 2008 par l'Etat, une trentaine d'œuvres se répartissent dans les différentes stations de Santiago : "Géométria andina » de Ramón Vergara réalisé en 1993 fut l'une des premières œuvres artistiques à entrer dans les sous-sols de la ville, stratégiquement situé à la station centrale de Los Leones. L'œuvre "Chile Hoy» du peintre Guillermo Muñoz Vera décline en 14 tableaux des vues de paysage et de la population du Chili sur les quais de la station de La Moneda. Le site internet de la corporation argumente ce choix : son œuvre "reflète le paysage et l'esprit de tout le Chili. Ce sont des images originales qui reflètent l'ampleur et la particularité de chaque lieu $»^{2}$. 


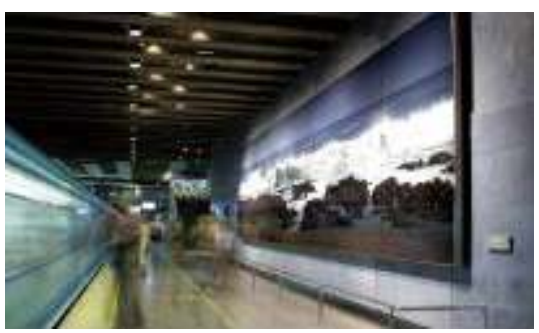

Station La Moneda

Photo de Thomas Wedderville (c) pour le bulletin Metroarte cultural corporation $n^{\circ} 7$

Geométrica andina, 1993, Ramón Vergara Grez, station de métro Los Leones

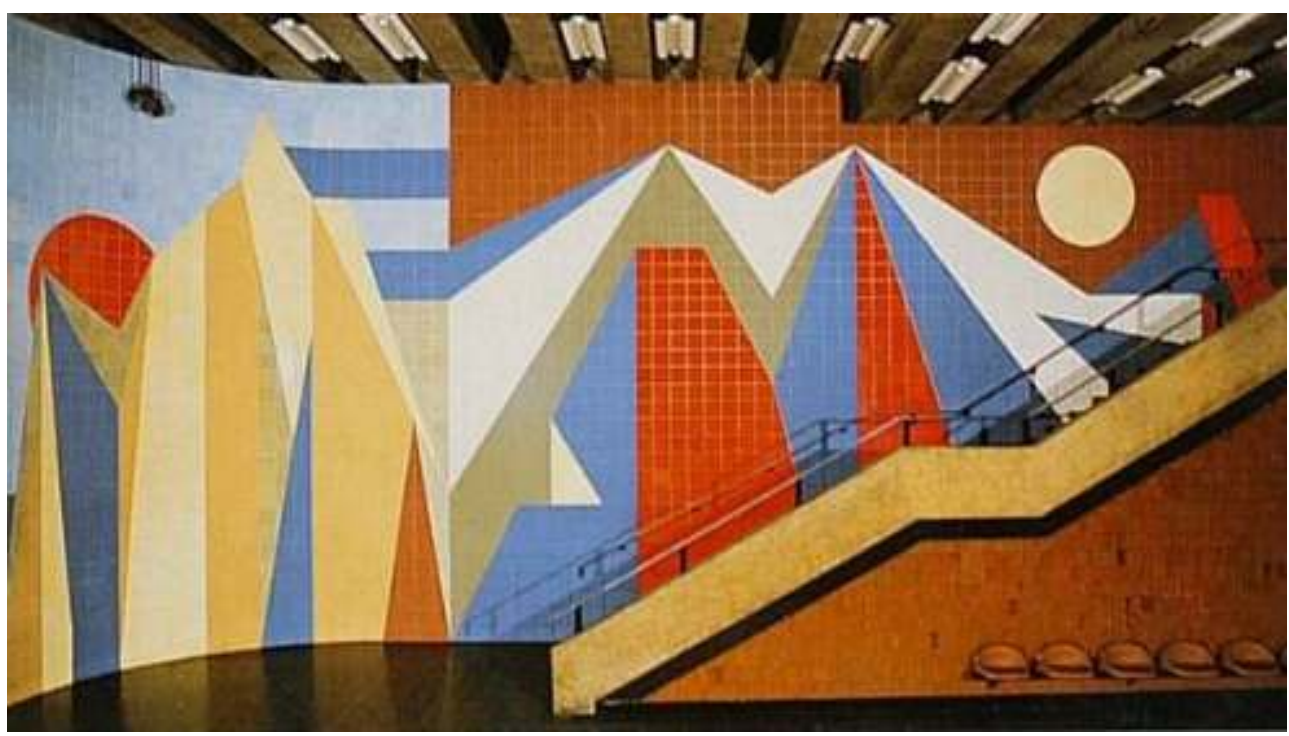

On ne manquerait pas d'évoquer le caractère particulier que tient la nature comme marqueur identitaire de la nation; comme le défend Sergio Villalobos-Ruminott «tout au long du XIXème siècle, la nature indomptable de la pampa, la forêt et le désert apparurent comme limites du projet de civilisation latino-américain et comme marque indélébile de notre passé culturel »(VILLALOBOS-RUMINOTT, 2008: 15-49). Mais cette adhésion presque spontanée entre un pays et ses paysages singuliers, nous ferait presque oublier que l'association entre identité nationale et image de la nature est davantage le fruit d'une construction historique et culturelle.

L'œuvre de José Venturelli, «Chile ( (1972) disposée à l'entrée de la salle des Arts Visuels $\mathrm{du}$ tout récent Centre Culturel Gabriela Mistral (GAM) ouvert en 2010, offre un bon exemple: l'environnement que nous assimilons sans hésitation à une nature représentative du Chili - comme le suggère son titre - plantent le décors d'une histoire prête à se dérouler. Mais aurait-on fait cette démarche déductive sur fond de logique, si le tableau nous avait été donné à voir sans son titre, dans un autre lieu et à une autre époque? 
Chile, José Venturelli, 1972, 250x900 cm.

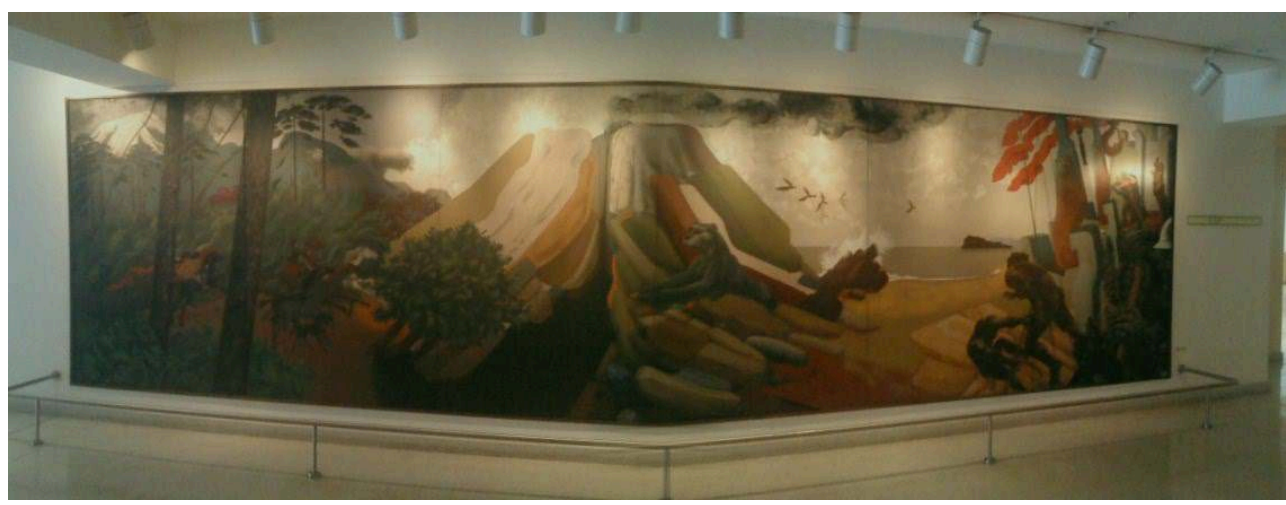

Tableau anciennement situé au Centro de Convenciones dans le même bâtiment, siège de la UNCTAD en 1872 et renomé « Diego Portales » par le gouvernement dictatorial.

GAM, entrée de la salle des Arts Visuels

L'interprétation, comme le souligne Bruno Péquignot ${ }^{3}$, dépend encore de la culture et de l'inconscient de celui qui regarde, et de sa capacité à reconnaître quelque chose dans une image, poursuivant ainsi les travaux de Francastel : «à partir du moment où toute vision est dans le temps, aucune image ne se forme sans participation de la mémoire collective. [...]. Dans toute image, il y a la rencontre et trace à la fois d'un phénomène et d'une conscience" (Francastel, 1970: 61). La capacité de s'entendre sur des codes de représentation dépend d'une culture commune à travers le partage d'un imaginaire collectif. Les courants abstraits en peintures rompant avec la figuration n'ont fait que rendre de plus en plus visible le travail de construction que suppose tout œuvre artistique. Mais encore faut-il, comme le suggère le sociologue Howard Becker ${ }^{4}$, poser comme modalités de l'interprétation - en plus du contexte social, historique, et culturel- les différents mécanismes qui régissent le champ artistique; loin d'être un ensemble unifié, celui-ci se décline en une variété de mondes composés d'interactions incessantes entre différents acteurs (producteurs, intermédiaires, mécènes, exposants, publics, ect...) qui vont inscrire les productions artistiques non pas comme des œuvres finies, mais comme des éléments intégrant un certain parcours.

D'autre part, la Nation en tant qu'idée abstraite, mobilise toute une série de représentations autour de mythes fondateurs et de figures mobilisatrices qui vont souvent bien au-delà des orientations politiques énoncées par un gouvernement ${ }^{5}$; ses contenus soumis à un perpétuel remaniement peuvent ainsi donner une approche intéressante quant à la délimitation du territoire naturelle en même temps que du territoire artistique. L'empreinte de la nature rend ainsi suspecte tout attention à son égard : comment va se faire ce rapprochement, quel impact cela va-t-il avoir dans le monde des arts, et en quoi cette problématique vient s'imbriquer à la définition d'une identité nationale?

6 A travers les différentes réformes et interventions politiques engagées dès la prise de pouvoir du Général Pinochet, nous tenterons de voir comment l'invocation et l'évocation de la géographie du Chili a été particulièrement forte et constante sous la dictature militaire (1973-1990). La nature en tant que thème artistique s'inscrit dans une large tradition picturale et devient objet d'ajustement. On pourrait alors se demander dans quelle mesure les images de la nature interviennent ou non dans la construction de l'image de la nation, la valeur que l'état lui accorde, et sa place 
notamment par l'instauration de nouvelles politiques de visibilité (concours, expositions, commandes) qui vont influer sur la réception de ces œuvres et leurs significations suivant leur contexte d'émergence. Cette politisation des circuits institutionnels de l'art viendra également conforter la notoriété de quelques artistes paysagistes et nous rappelle que toute notion de jugement et d'évaluation se situe au cœur même de l'action artistique.

\section{Restauration nationale et patriotisme}

7 Après le coup d'état du 11 septembre 1973, le nouveau gouvernement civil et militaire va instaurer un autoritarisme fondé sur l'union de deux systèmes de valeurs sans précédent dans l'histoire du pays: la sécurité nationale et le marché néolibéral ${ }^{6}$. Les premières vagues de violence et de répression à l'encontre des artistes soupçonnés d'avoir soutenu le gouvernement d'Allende, vont conduire nombreux d'entre eux sur le chemin de l'exil ou de l'emprisonnement. Le chanteur populaire Víctor Jara torturé et assassiné à l'Estadio Nacional deviendra le symbole de cette violente répression envers les artistes. Le gouvernement soutiendra tout au long de son mandat une politique culturelle parfaitement définie et cohérente ${ }^{7}$, s'enracinant essentiellement sur l'élimination du lègue socialiste et communiste, et la restauration de l'unité nationale ${ }^{8}$. L'urgence de l'affaire s'illustre dans le premier paragraphe de l'Acte de Constitution de la Junte du Gouvernement, dicté le jour même du coup militaire. Cet acte établit "l'engagement patriotique de restaurer la "chilenidad" " avec en filigrane l'idée que "lo chileno » se rencontre dans la trame historique et culturelle du pays.

8 Les récentes investigations sur cette période montrent comment le régime militaire a tenté d'éliminer les expressions culturelles mises en avant par l'Unité Populaire, allant jusqu'à changer le nom des rues, la perception, et la configuration esthético-sociale de la ville ${ }^{10}$ : l'exemple le plus frappant demeure le Stade National et le Stade du Chili convertis en centre de torture et de réclusion durant les premiers mois du régime. S'ajoute à cela les nombreuses opérations de nettoyage des murs de la ville : ainsi de grandes fresques réalisées par différents collectifs d'artistes - appartenant pour la plupart aux partis de la gauche comme la Brigade Ramona Parra, Brigade Elmo Catalán, Brigade Inti Peredo pour les plus célèbres - seront tous recouvertes de peinture blanche. Ces brigades, autrefois mises à l'honneur sous le gouvernement de Salvador Allende seront inlassablement stigmatisées par la Junte militaire qui les contraindra à agir dorénavant en toute clandestinité. L'intention était ainsi d'initier un processus d'éradication de n'importe quel indice, symbole, ou réminiscence associée à la période de l'Unité Populaire (GUILLAUDAT-MOUTERDE, 1998). Dans le journal national El Mercurio on peut lire que les forces armées sont allées jusqu'à enlever une statue du Che Guevara dans la commune de San Miguel. Cette nouvelle zone consensuelle va passer par un contrôle rigoureux des médias, de la presse, radio, télévision, universités, bibliothèques et musées. Les anciens directeurs seront remplacés par de nouveaux dirigeants principalement issus de l'armée.

9 La seconde mesure importante concerne les campagnes de restauration, et diverses initiatives destinées à délimiter un patrimoine culturel. En 1974, le tout nouveau Conseil Culturel de la Junte du Gouvernement et le Département Culturel du Secrétariat Général du Gouvernement soulignent la nécessité de renouer avec l'héritage du pays. Le but de ces campagnes est de "réparer la situation de décadence nationale reflétée par la 
perte de l'identité et du sens de nation, le manque d'une conception géopolitique stratégique de l'Etat, l'imitation de ce qui vient de l'étranger, la négligence vis-à-vis de notre histoire et de ses héros, en définitive, la disparition quasi-complète du « ser nacional $»^{11}$.

10 Ces expressions, comme le montre Munizaga ${ }^{12}$ sont fréquemment utilisées dans les discours du Pinochet (MUNIZAGA, 1988), et préfigurent la mise en place de tout un travail ambitieux autour de la reconstruction de la patrie. C'est dans cette perspective que la Direction du Tourisme, la Corporation de Réforme Agraire (CORA) et le Collège d'Architecture du Chili vont se concentrer sur l'annexion et la conservation des vieilles maisons de campagne et des fermes «typiquement » chiliennes. Dans le même temps tout un éventail d'initiatives et d'activités culturelles vont prendre place dans les grands centres artistiques et s'articuleront en majeure partie autour de la redécouverte des grands maîtres de la peinture chilienne, de l'artisanat, et de la beauté du territoire national: on en trouve une parfaite illustration au travers de l'exposition « Peintures et sculptures pour la reconstruction » en octobre 1983 au Musée National des Beaux-Arts, mais aussi bien plus tôt lors du "Premier Salon National d'Artisanat " (novembredécembre 1973) ou encore, l'exhibition au titre évocateur « Précurseurs étrangers de la peinture chilienne » (1974) au Centre Culturel de Las Condes.

11 Ainsi le gouvernement militaire encourage un resserrement autour des frontières du pays, substituant à la culture nationale, la culture de la nation. La rupture apparait d'autant plus brutale que le gouvernement de l'Unité Populaire par ses nombreuses réformes structurelles, prônait une démocratisation des arts étendus à toutes les couches sociales notamment par le biais de diverses expositions itinérantes. On voit alors naître l'idée d'une nation chilienne liée par un passé et une destinée commune avec les autres pays du continent. En témoigne l'inauguration d'un Institut latinoaméricain à la Faculté de Chili. A contrario du gouvernement du général Pinochet, l'Unité Populaire s'incarnait à travers une identité nationale dont le peuple en était l'épicentre et la force majeure. Dans son discours d'inauguration du Musée de la Solidarité, Salvador Allende encourageait la participation des artistes au devenir de « l'homme nouveau » et à toute forme de créativité : l'envoi de la Brigade Ramona Parra à la Biennale de Paris en 1972 pour représenter la section chilienne en est peut être le meilleur exemple. La politique du Général Pinochet abandonnera la trajectoire de son adversaire pour substituer au peuple chilien comme fondement de l'unité nationale, le territoire chilien, sa richesse et sa diversité. Il gardera jusqu'à la fin de son gouvernement le souci de mettre en avant des «images de notre territoire réellement attractives, [...] qui reflètent de beaux lieux maritimes, paysages de cordillères et urbains $»^{13}$.

12 Nous voyons donc comment cette construction particulière d'un système de perception a fortement privilégiée certaines tendances ou thématiques artistique tout en en excluant d'autres (théâtres populaires, musiques folkloriques, art urbain). Cette position du gouvernement ne fera qu'accroître une méfiance et une distance critique vis-à-vis des circuits officiels de la part des nouveaux artistes émergents, notamment après la fermeture forcée d'expositions emblématiques comme celles de Guillermo Nuñez à l'Institut Franco-Chilien en 1974.

\section{Concours nationaux et politique de visibilité}

13 Les nouvelles restructurations engagées par le régime vont donc avoir un impact conséquent sur le monde de l'art à travers l'organisation d'une multitude de concours 
nationaux qui vont favoriser ainsi la notoriété de quelques artistes. Par ailleurs la peinture et la sculpture maintiennent tout deux leur présence sur la scène nationale comme medium traditionnel, car leur production furent depuis toujours privilégiées dans les centres universitaires et dans les formations artistiques (IVELIC, 1990: 205-224); il faut savoir que l'université a été pendant longtemps le centre de production et de diffusion de l'art au Chili et que le Musée Nationale des Beaux-Arts faisait office d'autorité en matière de goût et de tendance.

Après le décret de loi de 1978 n. 2396 établissant l'augmentation des prix nationaux, le Musée Nationale de Beaux-Arts abritera de nombreux concours de peinture organisés par la Junte avec le soutien d'entreprises privées. En 1981, l'entreprise Chiletabacos finance un premier concours de peinture au Musée National des Beaux-arts ayant pour thème "Reencuentro pictórico con Chile : nuestra cordillera». Le premier prix fut décerné à Robinson Mora, ancien élève de l'école des beaux-arts se revendiquant de l'abstraction géométrique, avec son tableau Panorama Cordillera AB-8. En 1975, ce même artiste avait obtenu la mention honorable au concours sur l'Arbre organisé par le Musée d'Art Contemporain, et remporté en 1979 le grand prix du Concours de Peinture du Ministère des Relations Extérieures. Ses œuvres, comme il l'affirme, s'interrogent sur la perception individuelle de la nature existant dans le subconscient de l'homme. Mais ici l'empreinte particulière de la Cordillère occupe une fonction bien particulière. José Maria Palacios, l'organisateur de la manifestation s'explique :

"¿Por qué reencuentro y nuestra cordillera?

La idea fue reivindicar nuestro entorno como motivación artística. El paisaje chileno posee varias singularidades y algunas se han descuidado, sea porque no hubo estímulo o porque las corrientes de vanguardia las desplazaron.Se eligió la Cordillera como punto de partida, porque ella está ante nuestra vista en forma constante a lo largo de todo el territorio. Es nuestra columna vertebral. [...] a la Cordillera la vemos y la sentimos. Como efecto, no está sólo su presencia física sino también una sugestión de individualidad. La Cordillera nos protege, nos aísla, nos obliga a establecer una distinción de nosotros mismos » (MNBA, 1981 : s/p). 

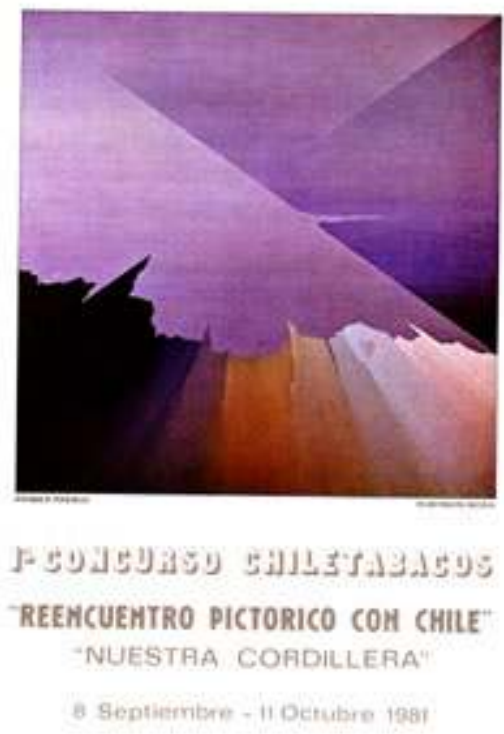

MUSEO MRCIOHAL DE BELIAS ARTES

Musée National des Beaux-Arts, 1981. Reproduction de la peinture lauréate de Robinson Mora, Panorama Cordillera AB-81, 1981.

La Cordillère des Andes sera un élément récurrent dans le choix des thématiques autant pour les peintres paysagistes que pour les organisateurs des concours. Toujours sur ce thème, le peintre Patricio de la 0 avait remporté le 3ème prix au concours de peinture organisé par le Ministère des Relations Extérieure en 1980 avec sa série "Pintura del regreso", deux ans après son retour au Chili. Cet artiste exposa régulièrement entre 1980 et 1990 dans diverses galeries et musées. Elève du célèbre paysagiste Pablo Buchard et de José Balmes, Patricio de la $\mathrm{O}$ affirmera tout au long de sa carrière son héritage avec la tradition paysagère chilienne, même s'il instaure une relation nouvelle dans ses peintures qui ne reposent ni sur l'observation directe du paysage, ni sur la construction d'un paysage mythifié; lorsqu'il réalise en 1981, son immense triptyque "Viva la cordillera de Los Andes» (qu'il exposera trois ans plus tard à la Galería Arte Actual), c'est à partir de photographies piochées dans les journaux, qu'il travaille; il les recompose, leur donne une nouvelle profondeur afin de représenter une cordillère qui fait partie selon lui de «cette inconscient visuel collectif». Le critique d'art Justo Pastor Mellado insiste sur sa capacité à rénover la peinture de paysage à partir d'outils de son époque, tout en questionnant le régime visuel de l'artiste du XXème siècle : "parce que le "vrai " paysage chilien est le paysage constitué par ces revues et non par la peinture [...] Le problème réside dans la constitution social d'une idée du paysage " (MELLADO, 1986). C'est donc en inversant le régime d'observation de l'expérience romantique que l'artiste invente un langage iconique qui condamne le mythe de l'observation directe de la nature. 
Tryptique El rey del Bosque, Patricio de la 0, 1982

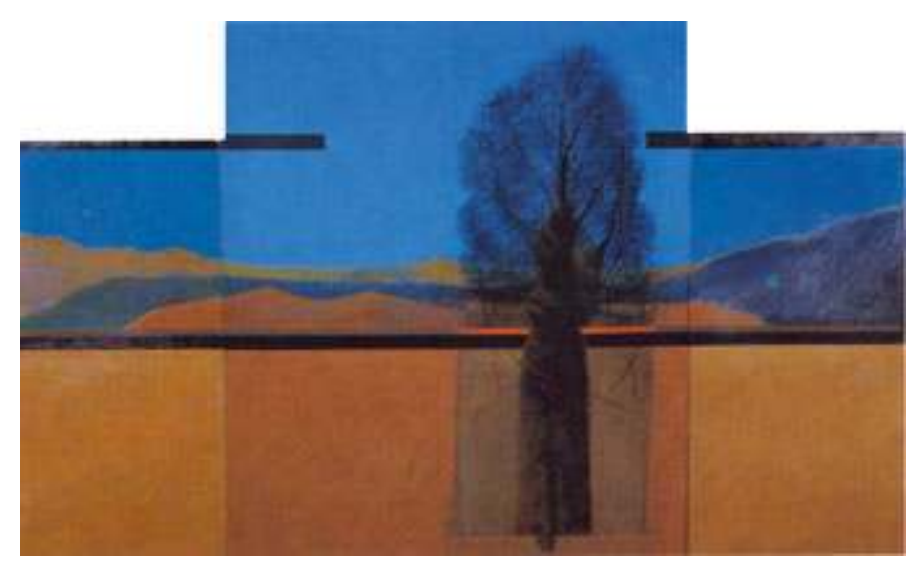

Museo Nacional de Bellas Artes

16 L'historien de l'art Milán Ivelic y voit quant à lui une rupture fondamentale avec les paysagistes traditionnels: "Il n'observe pas ni ne peint in situ à la manière des impressionnistes. Il n'y a pas de réflexion sur le paysage depuis sa contemplation, mais il pense son travail à partir de son travail lui-même; c'est-à-dire des procédés et méthodes employés pour peindre » (BADAL, 1998 : 39). Tout au long de sa carrière Patricio de la $\mathrm{O}$ a travaillé sur les différents paysages régionaux (désert, bords de mer, forêt, vallées, glaciers, etc...), mais son tableau « norte centro sur » dans lequel la cordillère unit les trois parties du tableau, ne peut que nous faire penser à cette "colonne vertébrale » allégorique évoquée plus haut par José María Palacios.

17 En prenant toujours la nature comme thème fédérateur, le Musée National des BeauxArts organise en 1982 le concours intitulé «El árbol en la pintura chilena » en partenariat avec le ministère de l'Education et le Département d'Extension culturel. Le Premier prix fut attribué à Carlos Maturana, plus connu sous le nom de Bororo, avec son œuvre expressionniste "Paisaje con árboles». Ancien élève de la Faculté des BeauxArts, Bororo exerça comme professeur entre 1975 et 1982 à la Faculté d'Art de l'Université du Chili. Reconnu pour son travail expressionniste, il remportera en 1988 le premier prix au concours «Paisaje Urbano » avec son œuvre "El centro de Santiago». Par ailleurs, ce concours sur l'arbre fut accompagné d'une rétrospective sur les grands maîtres paysagistes nationaux : on pouvait y voir entre autres les peintures de Alfredo Araya (1893-1954), Pablo Buchard (1875-1964) Rafael Valdés (1883-1923) et Pedro Lira (1845-1912).

18 Cette mise en scène et valorisation de quelques peintres de paysage contemporains concerne également Israel Roa, connu pour ses aquarelles et ses peintures de paysages chiliens. Il remporte sans surprise en 1985 le prix national de peinture. Cet artiste a pu exposer régulièrement dans divers endroit du pays, notamment à la Galerie Enrico Bucci, à la Galerie de Talca en 1974 et 1975, et au Musée d'Art Contemporain en 1978 et 1980. Elève de Juan Francisco González, on lui reconnaît une certaine habilité à saisir l'essence des paysages avec beaucoup de lyrisme. Avant sa nomination de 1985, l'écrivain Luis Oyarzún ira jusqu'à y voir une certaine «chilenidad » qui ressort dans ses tableaux, ce qui ne pourra faire de lui, qu'un peintre « digne » de rejoindre le palmarès des artistes nationaux comme le souligne le catalogue de l'exposition de la Galerie Enrico Bucci en 1977. Ce même critique le félicite de ne pas faire seulement de la montagne le discours sur le paysage chilien, mais d'être au contraire un explorateur du 
pays entier ${ }^{14}$. Ses peintures sur la diversité de la nature chilienne font écho aux aquarelles de Hardy Wistuba Stange. Ce dernier avait remporté en 1981, le premier prix $\mathrm{du}$ concours «Pintando Providencia» (à l'Instituto Cultural de Providencia) et a également réalisé une multitude de différents paysages chiliens, et en particulier ceux de la région de Puerto Montt, sa ville natale.

Enfin, il nous faut signaler les nombreux concours nationaux (entre 1985 et 1988) organisés plus particulièrement par l'Armée Navale, l'Armée de Terre et l'Armée de l'Air avec le soutien de la Fondation Nationale de la Culture et la Direction Générale de Mobilisation Nationale. Ces concours prennent pour sujet les grandes batailles de l'histoire du Chili. Ici, dans le cadre de la retranscription d'une histoire mythifiée, la chaine de montagne occupe une place bien particulière au point de composer dans certains cas, l'essentiel du tableau, et le plus souvent, le décor principal : lors du 3ème concours de 1987 sur le thème de l'Indépendance du Chili (1810-1823), Pablo Miquel Wotherspoon, représente la bataille de Maipú dans laquelle les personnages se perdent dans l'immensité des étendues montagneuses. Luis Cardenas Rojas avec son tableau « Hacia el triunfo y la gloria » figure la traversée de la cordillère par des soldats épuisés par la rigueur du relief et du climat. Réminiscence d'un genre pictural ancré dans une tradition ancienne, les peintures de paysage vont de plus en plus être dénigrées par les nouveaux artistes émergents, qui voient dans ces peintures un décalage avec les préoccupations actuelles du pays.

\section{Image de la nation : rejet du genre, modulation du paysage}

En effet, à partir des années 80 , la scène artistique émergente, qui prendra le nom de Escena de Avanzada sous la plume de Nelly Richard, va manifester une critique acerbe à l'égard du système institutionnel. D'autre part, les collectifs muralistes se reforment et de nombreux artistes exilés rentrent au pays. Une nouvelle dynamique artistique va se mettre en place autour d'une production alternative et dissidente, fondée sur la nécessité urgente "d'une culture avec une vraie identité nationale » (CATALÁN,1982:s/p) Dans son ouvrage Márgenes $e$ Instituciones Nelly Richard pointe l'instrumentalisation faite par le gouvernement à travers "la catégorie universalisante du beau avec son spiritualisme transcendant qui nie la matérialité sociale et politiques des signes artistiques " (RICHARD, 2007 [1987] : 122). Ce mécontentement et cette critique grandissante vis-àvis du système artistique chilien sera le moteur de la grande exposition organisée en 1987 à Madrid par le Ministère de la Culture d'Espagne, sous le titre explicite "Chile vive : memoria activa ». Un article du sociologue José Joaquín Brunner fait le point sur la situation :

Mi primera reacción es esta: Chile no tiene una sola identidad. No hay algo así como un "ser nacional", una imagen arquetípica de "lo chileno", una figura de lo nacional. De hecho, la retórica patriótica es entre nosotros mal vista y peor recibida. La escuchamos con escepticismo, nos suena hueca, a lo más le atribuimos un valor instrumental (ANTUNEZ et GUTIERREZ, 1987 : 9-12).

C'est dans ce décalage entre l'image d'une nation évoquée à travers les institutions culturelles, et les nouvelles propositions artistiques en lien étroit avec les changements socio-politique, que s'enracine la critique : 
«Como país no nos determina tampoco la naturaleza. Es falso que seamos una isla, un fin de mundo, una nación reconstruida cien veces sobre las ruinas de los terremotos. Esos son, en el mejor de los casos, nuestros "lugares comunes"; espacios simbólicos de entre los cuales algunos han querido inscribir los rasgos del "carácter chileno» (ANTUNEZ et GUTIERREZ, 1987 : 9-12).

L'exposition sera qualifiée par Enrique Campos, ambassadeur du Chili à Madrid, de «partielle et doctrinaire $»^{15}$. Cette rupture avec le gouvernement, mais aussi au sein même de la production visuelle, va se faire ressentir à travers l'attirance pour de nouveaux sujets artistiques comme la ville et le corps, de nouveaux médiums comme la photographie, la vidéo, les performances et les happenings, sous couvert d'une critique virulente contre l'académisme. Dans cette reformulation conceptuelle, la relation du peintre avec son environnement naturel occupe une place bien particulière :

La atracción por la naturaleza fue la motivación fundamental de los pintores chilenos ; su utilización como tema marcó su historia durante un siglo. Fue el punto de partida de la exploración del lenguaje pictórico que se apropió del mundo real, de la naturaleza física (GALÁZ et IVELIC, 2009 : 28).

Une des principales caractéristiques de la Escena de Avanzada sera de détruire les frontières entre les genres et les disciplines artistiques puisqu'elles impliquaient un certain ordre de la tradition canonique. C'est donc toute une stigmatisation du système de restriction et de prohibition des circuits institutionnels de l'art qui est pointé du doigt. Pour les nouveaux artistes comme pour les critiques, il s'agit dorénavant de "désobéir aux assignations formelles conventionnelles qui fixe la tradition artistique et littéraire du format-cadre (tradition pictural) au support-paysage (la matérialité vive du corps social)" (RICHARD, 2007 : 34). On assiste alors à une configuration inédite dans le rapport de force entre la politique du pays, et une nouvelle politique des arts qui marquera la singularité de cette période : Milan Ivelic ne manque pas de montrer cette rupture en comparant la période de l'Unité Populaire avec celle du régime militaire: "jusqu' à l'apparition du gouvernement autoritaire, la relation entre le champ politique et le champ artistique se déroulait sans conflits ». (IVELIC, 1990 : 205-224).

Cette autocritique de la tradition artistique chilienne ne cessera de croître et de chercher d'autres formulations au-delà des énoncés académiques : pour Nelly Richard, les artistes doivent chercher de nouvelles références plastiques aussi bien dans la manière de concevoir une œuvre, que dans son contenu. C'est pourquoi la critique artistique privilégiera davantage les interventions urbaines du Groupe CADA, les vidéos-installations de Carlos Leppe, mais surtout les interventions faites dans la nature: une vidéo d'Eugenio Dittborn de 1982 enregistre l'écoulement de 350 litres d'huile brulé dans le désert de Tarapacá. La même année, à New York, la fumée d'un avion dessine dans le ciel un poème de Raúl Zurita. Enfin, avec beaucoup d'ironie, Carlos Altamirano propose une intervention visuelle en inscrivant en lettre majuscule devant l'image d'une réunion de famille " Panorama de Santiago (huile sur toile) de J. F. González ». Le titre fait référence à un tableau du célèbre peintre Juan Francisco González (1853-1933). Peintre romantique et bohème, il était considéré comme l'un des quatre grands maîtres de la peinture chilienne du XIXèm siècle à côté de Pedro Lira, Alfredo Valenzuela Puelma, et Alberto Valenzuela Llanos. Ses œuvres étaient exposées au Musée National des Beaux-Arts de Santiago. D'autre part, le terme de "panorama » fait allusion au Chili a une activité d'oisiveté. Ces artistes vont ainsi travailler sur la déconstruction des modèles anciens en revendiquant un nouveau rapport au monde qui annihile toute logique de représentation. 
«Panorama de Santiago (óleo sobre tela) J. F GONZÁLEZ », Carlos Altamirano, Intervention télévisuelle, 1982

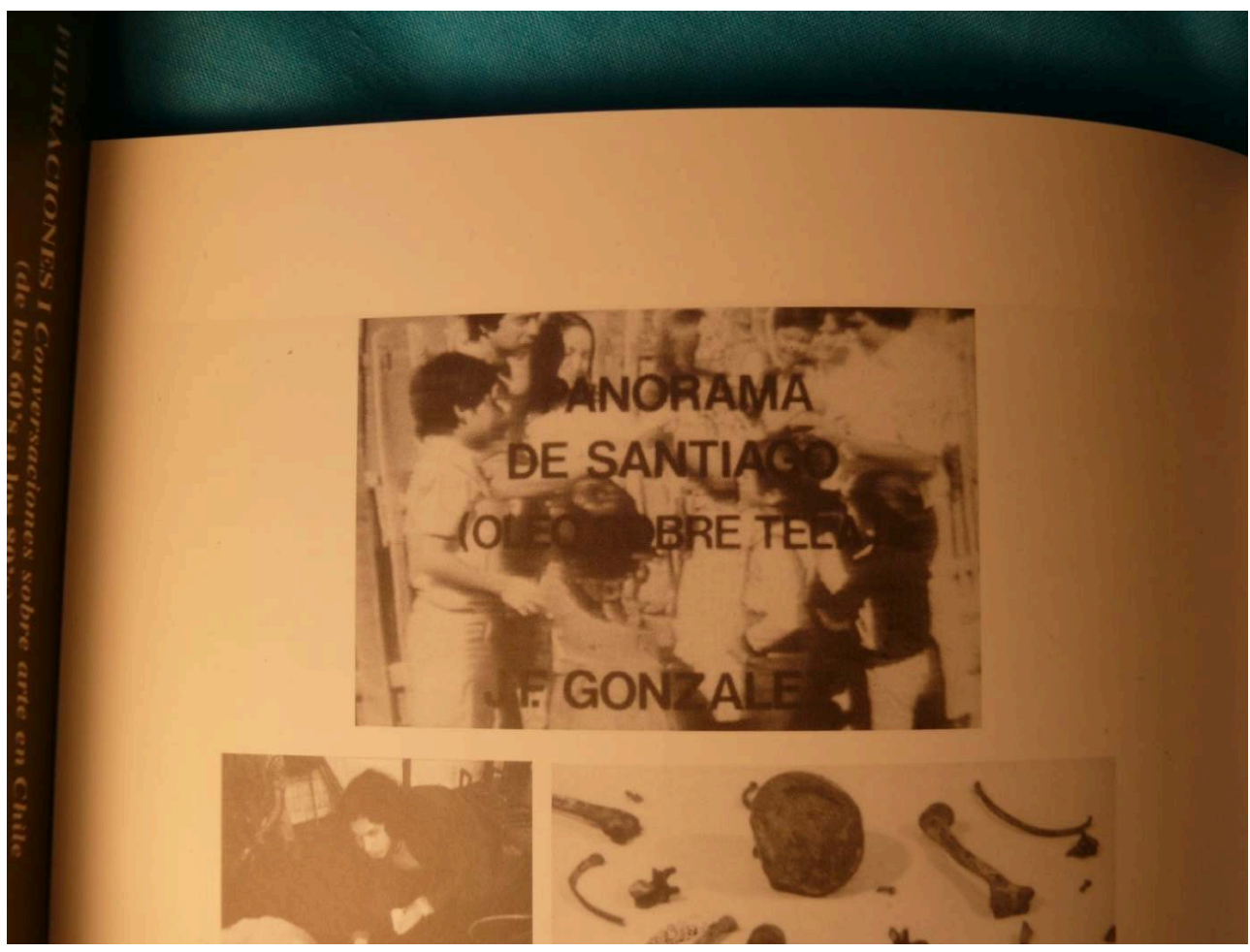

Mais déjà dans les années 50 , on pouvait noter une mise à distance de la peinture de paysage chez certains artistes chiliens: les nouvelles conditions imposées par la dictature n'ont fait qu'accélérer et rendre plus visibles ces transformations. Dans le catalogue de leur première exposition le 26 septembre 1956, les membres du groupe Rectágulo affirment :

Cientos de años de pintura basada en una copia de la realidad exterior o una interpretación de ella han revelado al artista una naturaleza oculta, misteriosa, abstracta; una realidad que está más allá de lo inmediatamente conquistable. Esto explica el rechazo y hasta el desprecio que hacen de la naturaleza la mayoría de los artistas de nuestro tiempo (Apud in GALAZ et IVELIC, 2009).

D'autre part, Justo Pastor Mellado va défendre l'idée que la configuration du paysage chilien provient en grande partie de l'orbite sémantique de la poésie, mais surtout qu'elle est partie intégrante de la culture européenne diffusée par le biais de la Academia de Bellas Artes du Chili.

La representación pictórica chilena es un defecto de construcción europea en lo que corresponde a la política de la imagen identificadora del rostro y del paisaje (MELLADO, $2000: 141$ ).

Symbole d'un ordre ancien, le rapport du peintre à la nature implique également un certain type de relation à l'objet (imitation, représentation, exaltation) qui ne correspond plus aux problématiques invoqués par les artistes contemporains. L'œuvre de José Léon "Nuestro paisaje- Mi paisaje » (1982-1984) illustre ce déplacement de la relation entre l'artiste et son environnement. L'artiste réalise sur un mur une composition de divers éléments : l'image d'un terrain de foot, des portraits d'artistes comme Raúl Zurita, Roser Bru, le célèbre personnage de bande-dessiné Condorito, la Cordillère des Andes. Dans la Série En tierra, José Balmes travaille quant à lui à partir de 
la terre qu'il étale sur ses toiles. Gaspar Galáz montre comment subsiste la volonté de l'artiste de rendre visible sa notion de paysage, non plus représenté, mais pourtant bien là : "ce paysage autre, cette désarticulation de la méthodologie paysagiste dans laquelle se présente l'objet et le modèle de cette pratique: la terre [...] la référence au paysage humain et naturel, est planifié comme plan de travail, explicitant les aspects figuratifs seulement à travers quelques éléments qui viennent synthétiser la vision qu' a l'artiste du monde, et plus précisément du nôtre» (GALÁZ, 1989).

\section{Conclusion}

La relation entre nature et nation sera donc particulièrement intense entre les années 80 et 90 , à la fois imprégnée par les réformes artistiques engagées par les générations précédentes, mais aussi marquée par un régime visuel particulier dessiné par le régime militaire. Les nouveaux critiques artistiques chercheront à mettre fin à une relation particulière qu'impliquait toute représentation du paysage. C'est pourquoi le rejet du thème de la nature dans les arts plastiques impliquait au-delà du simple abandon iconographique, la volonté de rompre avec une peinture aristocratique et bourgeoise ; on pourrait presque y voir la volonté d'abandonner le système d'un art pour l'art uniquement dédié à la contemplation personnelle. Les ruptures soulignées avec les contenus antérieurs de la peinture académique n'en deviennent que de plus en plus significatif dans le contexte de cette période. Mais si la nature fascine encore et toujours, sa relation avec les politiques gouvernementales n'en devient que plus captivante : "Si l'œuvre est d'abord travail, elle est, comme tout travail, transformation d'une nature. Mais quelle nature? Et transformée dans quel but? C'est un grand mot lâché que celui de nature. Nombre d'artistes n'ont que lui à la bouche» (PASSERON, 1986: 10). Maintenant interrogeons-nous : ces récentes peintures habillant les quais du métro auraient-elles la même signification si elles avaient été placées au Musée des Beaux-Arts?

\section{BIBLIOGRAPHIE}

ANTÚNEZ, N. et GUTIÉRREZ, P. (1987), Chile Vive : memoria activa. Santiago, Céneca e Instituto de cooperación iberoamericana.

BADAL, G. (1998), Ventana al paisaje Patricio de la O. Santiago, Ed. Ocho Libros.

BRUNNER, J. J. (1985), A propósito de Políticas Culturales y Democracia. Un ejercicio formal. Santiago, Programa FLACSO, n. 254.

BRUNNER, J. J. (1990), « La cultura chilena durante la dictadura » in Revista Cuadernos, Hispanoamericanos, Santiago, agosto / septiembre.

CATALAN, C. et MUNIZAGA, G. (1986), Politicas culturales estatales bajo el autoritarismo en Chile. Santiago, Centro de Indagación y Expresión Cultural y Artística.

FRANCASTEL, P. (1970), Etudes de sociologie de l'art. Paris, éd. Gallimard. 
GALAZ, G. (1989), « Nuestra patria historia a nuestra tierra », in En tierra (a 50 años de Winnipeg). Santiago, Galería Plástica Nueva.

GALAZ, G. et IVELIC, M. [1988] (2009), Chile Arte actual. Ed. Universitarias de Valparaíso.

GALENDE, F. (2007), Filtraciones 1. Conversaciones sobre arte en Chile (de los 60's a los 80's). Santiago, ARCIS, Ed. Cuarto Propio.

GUILLAUDAT-MOUTERDE, P. (1998), Los Movimientos sociales en Chile 1973-1993. Santiago, Ed LOM.

ERRAZURIZ, L. H. (2009), « Dictadura militar en chile antecedentes del Golpe estético-cultural », in Latin American Research Review, vol. 44, n. 2.

IVELIC, M. (1990), «Itinerario de las artes visuales », in Cuadernos latinoamericanos, Santiago, agosto - septiembre.

MANDOKY, K. (2006), Prácticas estéticas e identidades sociales. México, Ed. Siglo XX.

MELLADO, J. P. (2000), Cuadernos de la Escuela de Arte, textos estratégicos, n. 7, octubre, Santiago, Ed. Pontificia Universidad Católica de Chile.

MELLADO, J. P. (1986) « Nota sobre la pintura de Patricio de la O ». In: Catalogue de l'exposition Pinturas 1985-1986, Santiago, Galería Arte Actual.

MUNIZAGA, G., (1988), El discurso público de Pinochet: un análisis semiológico. Santiago, Ed. Cesoc/ Céneca.MUSEO NACIONAL DE BELLAS ARTES, MNBA (1981), Catalogue de l'exposition « Concurso Chiletabacos », Santiago, 8 septembre-11 octobre.

ORELLANA, C. (1990), "La cultura chilena en el momento de cambio" in Cuadernos hispanoamericanos, n. 482-483, 1990.

PASSERON, R. (1986), L'CEuvre picturale et les fonctions de l'apparence. Paris, Ed. Vrin.

RICHARD, N. [1986] (2007), Márgenes e instituciones, Arte en Chile desde 1973. Santiago, Ed. Metales pesados.

VILLALOBOS-RUMINOTT, S. (2008), “Modernidad y dictadura en Chile: la producción de un relación excepcional" in A Contra corriente, University of Arkansas vol. 6, automne.

\section{NOTES}

1. Issue de la Corporation Culturelle fondée en 1992 avec le soutien d'entreprises privées, celle-ci organise aussi divers concours littéraires, possède un centre culturel dans la Station Quinta Nomal, en plus d'une galerie.

2. Extrait du site internet de la Corporation : http://www.metrosantiago.cl/cultura/metroarte/ catalogo

3. Professeur de sociologie des arts et de la culture à l'Université Paris III, Bruno Péquignot a notamment publié Recherches sociologiques sur l'image, éd. l'Harmattan, Paris, 2008, et Sociologie des arts : domaines et approches, éd. Armand Colin, Paris, 2009.

4. Howard Becker est un sociologue indépendant, héritier de la tradition de l'Ecole de Chicago. Après avoir pris comme objet d'étude le milieu de la musique jazz, il développe dans ses travaux une théorie interactionniste en réaction à la tradition fondamentaliste. Ses principaux ouvrages sont Outsiders, étude de sociologie de la déviance, ed. Métailié, Paris, 1985 (ed originale 1963), Les mondes de l'art, ed. Flammarion, Paris, 1981 et Comment parler de la société, éd. La Découverte, Mayenne, 2009. 
5. Je vous renvoie à l'ouvrage d'Anne-Marie Thiesse, La Création des identités nationales, éd. du Seuil, Paris, 1999. L'auteur pointe l'aspect construit des nations et des identités nationales depuis le XVIIIe siècle et se penche sur ce lien primordial qui ne lie plus la société à un prince ou à une personne physique, mais à une unité sociale abstraite qui est la nation. Voir aussi Benedict Anderson, L'Imaginaire national : réflexions sur l'origine et l'essor du nationalisme, éd. La Découverte, Paris 2006 et Eric J. Hobsbawm, Nations et nationalisme depuis 1780, éd. Gallimard, Paris 2001.

6. Voir les travaux du sociologue José Joaquín Brunner dont bon nombre de ses investigations portent sur les transformations sociétales et culturelles engendrées par la dictature. Notamment BRUNNER, $1990: 24$.

7. Thèse défendue dans ORELLANA, $1990:$ 49-54.

8. Pour approfondir la question de l'instauration du gouvernement totalitaire, voir Revista Atenea n. 428, publiée par l'Universidad de Concepción, immédiatement après le coup d'état de Pinochet. On peut d'autre part se référer aux prix nationaux octroyés pendant les premières années de la dictature où apparait clairement une politique culturelle assez explicite, sans compter sur le programme éditorial du journal national El Mercurio et son organe culturel, "Artes y Letras ». L'ouvrage de Joaquín Lavín (1986), La Revolución silenciosa. Santiago, Ed. Zig Zag, décrit tous les aspects culturels de l'implantation du modèle néolibéral de l'Ecole de Chicago.

9. Décret de loi n. 1.11 par Acte de Constitution de la Junte du Gouvernement, 11 septembre 1973. 10. Voir ERRAZURIZ, 2009. Cet article s'inscrit dans le cadre d'un plus ample projet d'investigation par FONDECYT N. 1060675, "Estética del gobierno militar. Estética de la dictadura" (2006-2008).

11. Asesoría cultura de la Junta de gobierno y del departamento cultural de la Secretaría General de Gobierno 1974 apud dans MUNIZAGA, 1988 : 37-38

12. Giselle Munizaga s'est penchée sur les phénomènes de communication en Amérique Latine et plus particulièrement dans le Chili de Pinochet en prenant pour objet d'étude la presse, la radio, la télévision. Elle travaille sur les manipulations des médias par les secteurs dominants.

13. In Mensaje Presidencial de 1978 (p. 443). Biblioteca del Congreso Nacional

14. Retranscription des écrits de Luis Oyarzún, « La naturaleza en Israel Roas », dans le catalogue de l'exposition "Israel Roa" à la Galería del Arte de Talca. Mai-juin 1990.

15. El País, Mardi 20 janvier 1987, retranscrit dans ANTÚNEZ et GUTIÉRREZ, $1987: 66$.

\section{RÉSUMÉS}

A travers l'instauration d'une vision très patriotique de la nation sous la dictature du général Pinochet (1973-1990), la tension entre image de la nation et son rapport à la nature s'est jouée audelà des problématiques de la scène artistique contemporaine. Si l'on tient compte de tous les acteurs qui participent aux circuits de production et de diffusion de l'art comme le propose la méthode d'analyse de Howard Becker, nous pouvons conclure à une visibilité constante des peintres paysagistes se revendiquant de la tradition des grands maitres chiliens. Pourtant, la critique artistique qui va se mettre en place sous l'impulsion de Nelly Richard à partir des années 80 rejettera la posture qu'implique toute représentation de la nature, afin de renouer avec les préoccupations socio-politiques du moment.

Con una visión muy patriótica de la nación bajo la dictadura del general Pinochet (1973-1990), la tensión entre imagen de la nación y su relación con la naturaleza sobrepasa las preocupaciones 
de la escena artística contemporánea. Si tenemos en cuenta todos los actores que participan en los circuitos de producción y de difusión del arte según la metodología de análisis de Howard Becker, nos damos cuenta de que los pintores paisajistas que se afiliaron a la tradición de los grandes maestros ocupan un espacio de visibilidad constante. Sin embargo, la crítica artística impulsada por Nelly Richard en los años 80 rechazará la posición que implica toda representación de la naturaleza, con el fin de enfrentar los problemas socio-políticos del momento.

\section{INDEX}

Mots-clés : nature, politique, Pinochet, concours, avant-garde, institutions

Palabras claves : naturaleza, política, Pinochet, concursos, Escena de Avanzada, instituciones

\section{AUTEUR}

\section{CARINE LEMOUNEAU}

Université Paris I Panthéon-Sorbonne et Universidad de Chile. ED 441 - Laboratoire HiCSA

Histoire Culturelle et Sociale de l'Art.

c.lemouneau[at]gmail.com 